\title{
COMPARATIVE ANALYZES OF TECHNOLOGICAL TOOLS BETWEEN INDUSTRY 4.0 AND SMART CITIES APPROACHES: THE NEW SOCIETY ECOSYSTEM
}

\author{
Wilson Gasparotto Storolli \\ FCA - UNICAMP, Brazil \\ E-mail: wstorolli@gmail.com \\ leda Kanashiro Makiya \\ FCA - UNICAMP, Brazil \\ E-mail: iedakm@gmail.com \\ Francisco I. Giocondo César \\ IFSP/FCA - UNICAMP, Brazil \\ E-mail: giocondo.cesar@gmail.com
}

Submission: 05/03/2018

Revision: 29/03/2018

Accept: 15/10/2018

\section{ABSTRACT}

Today the growth of modern cities is unprecedented in the history of urbanization and the urban environmental problems have also been increased. Unfortunately, we do not have much time to correct past failures, improve the status quo and ensure the protection of environment. Consequently, developing sustainable urban planning is important and its role in urban management issues is an objective that requires new approaches.

On the other hand, Industry 4.0 (I.4.0), also called the $4^{\text {th }}$ Industrial Revolution, carries impacts in the production in companies, as well as in the economy and society, with disruptive character, creating new markets and destabilizing the traditional way of doing business. Once $\mathbf{1 . 4 . 0}$ is a strategic approach to the integration of advanced control systems with internet technology, enabling communication between people, products and complex systems, it is expected to follow the same in the Smart Cities development. 
INDEPENDENT JOURNAL OF MANAGEMENT \& PRODUCTION (IJM\&P)

http://www.ijmp.jor.br

v. 10, n. 3, May - June 2019

ISSN: 2236-269X

DOI: 10.14807/ijmp.v10i3.792

This article aims to relate technological tools of $\mathbf{1 . 4 . 0}$ and the dimensions of "Smart Cities", based on an analytical framework for better understanding the emergence of new society ecosystem focused on the redefinition of the concept of cities, urbanism and way of life, motivated by this new reconfiguration.

The study has answered the questions: 1- Smart Cities are using common technological tools of I.4.0, and 2- I.4.0 Tech tools have relevance as main booster for "new" Smart Cities concept, giving a guidance to the managers for a strategic development orientation, suggesting they should prioritize the introduction or development of IOT, IOD and IoS.

Keywords: Smart Cities, Industry 4.0, Fourth Industrial Revolution

\section{INTRODUCTION}

The Industrial Revolution is a movement of humanity's evolutionary nature process (GERLITZ, 2015). The $4^{\text {th }}$ Industrial Revolution, being called "Industry 4.0", had its name coined in 2011 in Germany (originally Industrie 4.0), being part of the government strategy with industrial and academic societies to recover the world technological leadership and based on the complete interconnection of information in real time, using the concepts throughout the value chain of Internet of Things (IOT), Internet of Services (IoS), Big Data, Smart Factory, Cyber-Physical systems (CPS) etc. (KAGERMANN et al., 2013).

I.4.0, as an industrial revolution with disruptive character, more than carrying impacts in the economy and society, also brings challenges and opportunities in the scientific, technological, economic, social or political dimensions (ZHOU; ZHOU, 2015).

Smart City, according to European Smart Cities 4.0 (2015), Caragliu et al. (2009), Maier (2016), Roblek et al. (2016), Lom et al. (2016), Kummitha and Crutzen (2017), Ahvenniemi et al. (2017) and Basiri et al. (2017), Trilogy based on Technology, Government and Society, introduces the features of a Smart Economy, Smart Mobility, Smart Environment, Smart People, Smart Living and Smart Governance, in which the main goal is to ensure the sustainability of cities, improving the quality of life and security of their citizens, and provide maximum energy efficiency, using the contribution of the latest technologies, impacting on those six mentioned key areas. 
INDEPENDENT JOURNAL OF MANAGEMENT \& PRODUCTION (IJM\&P)

http://www.ijmp.jor.br

v. 10, n. 3, May - June 2019

ISSN: 2236-269X

DOI: 10.14807/ijmp.v10i3.792

The purpose of this article is, in an unprecedented way to the best of our knowledge, to identify the relations of the Smart Cities development dimensions approach with "Industry 4.0" revolution, its common technological tools, and the relevance on boosting a "new" Smart Cities concept, as a guidance to the managers for a strategic development orientation.

\section{THEORETICAL CONCEPTUAL FRAMEWORK}

\subsection{Industry $\mathbf{4 . 0}$}

The $4^{\text {th }}$ Industrial Revolution or Industry 4.0, originally called "Industrie 4.0", was identified in 2011 in Germany, during the development of the German Government Strategic Plan for 2020. According Gerlitz (2015), this worldwide movement is an evolutionary nature process and its definition comes naturally evolving, but basically is: "a strategic approach to the integration of advanced control systems with internet technology, enabling communication between people, products and complex systems (ANDERL, 2014)".

At that time, Germans recognized I.4.0 as if the world was starting a new industrial revolution, because of the disruptive technology character impacting the economy and society.

For this study, a literature review was conducted to identify the main factors necessary for the implementation of the concepts of "I.4.0", and the main technological tools cited in Smart Cities papers are: Additive Manufacturing-3D print(ANDERL, 2014), Automatic Guided Vehicle-AGV (MARTÍNEZ-BARBERÁ; HERRERO-PÉREZ, 2009), Application computer programs-APP/APPS (Waze, Uber, etc) (SANDERSON, 2010), Artificial Intelligence-AI (BROOKS, 1991), Automation System (PARASURAMAN et al., 2000), Big Data Warehouse (MILOSLAVSKAYA E TOLSTOY, 2016), Big Data Analytics (RUSSOM, 2011), Cloud Computing (MELL; GRANCE, 2011), Cyber-Physical System-CPS (KAGERMANN et al., 2013), Cyber Security (SOLMS; NIEKERK, 2013), Data Mining (HOPEN, 2015), Business Intelligence-BI (HOPEN, 2015), Horizontal Integration (KAGERMANN et al., 2013), Internet of Data-IoD (ANDERL, 2014), Internet of Services-IoS (SCHWAB, 2016), Internet of Things-loT (FLEICH, 2010), Simulation Software (BANKS, 1998), Smart Devices (phone, tablet, watch) (THOMPSON, 2005), Smart Factory (HERMANN et 
al., 2015), Smart Identification (RFID ${ }^{1}$ tag, NFC ${ }^{2}$, Bluetooth) (MAGLIO; LIM, 2016), Smart Sensing (PRYTULA, 2011), Traceability (METZNER et al., 2014; Machado, 2000), and Information and Communication Technology-ICT (SCHUMACHER et al., 2016).

Despite not being a specific I.4.0 technique, we have contextualized ICT to better understand some approaches in this study and the application in 1.4.0. We can consider a wide range of tools, such as: IoT, IoS, IoD, Cyber Security, Cloud Computing, Big Data \& Analytics, Data Mining, BI, etc. IoT requires highly scalable computing platforms that can manage the big loT data in terms of processing, access and storage without affecting the performance of the application (Ahmed et al., 2016).

To understand the thematic of industrial revolutions and how 1.4 .0 can impact on the afore mentioned environmental, social and economic dimensions changes, can contribute to give better directions in Smart Cities development.

\subsection{Smart City}

According to Anthopoulos (2016), the term "Smart City" was coined in 1994, at the launch of the "Amsterdam Digital City" in Holland with the Geneva-MAN project, involving the redefinition of that metropolitan area.

Basiri et al. (2017) mention that paying attention to the development of sustainable urban planning is important, and its role in urban management issues is an objective that requires a new approach to urban planning. The concept of smart cities already holds the potential to address aspects of the sustainability challenge by promoting citizens' participation, developing innovative and smart solutions for sustainability, increasing efficiency in city systems, and adopting a transparent and inclusive governance system.

Many definitions of smart cities exist, and a range of conceptual variants is often obtained by replacing "smart" with alternative adjectives, for example, "intelligent or digital". Intelligence has been basically associated with the ICT-based innovation, creating a "paradox" between "smart" and "habitable", because in some

\footnotetext{
${ }^{1}$ Radio Frequency Identification System

2 Near-Field Communication
} 
INDEPENDENT JOURNAL OF MANAGEMENT \& PRODUCTION (IJM\&P)

http://www.ijmp.jor.br

v. 10, n. 3, May - June 2019

ISSN: 2236-269X

DOI: 10.14807/ijmp.v10i3.792

cases there is no need to use technological intelligence, although tools are encouraging the leadership of attractiveness (ANTHOPOULOS, 2016).

According to him, several models of some authors evaluate Smart Cities using a series of definitions and indicators that address the urban and technological characteristics, and some of these organizations are drawing standards and homogenization of industrial products, among which the main ones are: 1International Standards Organization-ISO (2014) defines Smart City as a new concept and a new model, which applies new generations of ICT to facilitate the planning, construction and management of intelligent services. 2- International Telecommunications Union-ITU (2014) defines the Smart City as being one that is sustainable and innovative, using ICT and other tools to improve people's quality of life, the efficiency of the operation, urban services, and competitiveness, ensuring the interests of present and future generations, in the aspects of economic, social and environmental sustainability.

Any urban context that optimizes the provision of services through technology while keeping a balance between competitiveness and sustainability can be considered a "smart" city, town, village or neighborhood. A smart strategy aims to improve the quality of life of the people using the technology as a facilitator of this process. However, we must consider that information and technology alone will not build an intelligent city, but its capacity and ability to effectively and efficiently meet the needs of its citizens (CEBREIROS; GULÍN, 2014), and according to Kagermann et al. (2013, p. 5), I.4.0 tools will, undoubtedly, give direction and solve some current global challenges, such as resources and energy efficiency, urban production and demographic change.

Kourtit et al. (2017) say the advent of ICT has provided many opportunities to design and implement Smart and Sustainable Cities, sometimes also called Wired Cities (cities connected by cables and wires), intelligent cities, WIKI cities (from Wikipedia, used as a fast and light city), or digital cities. In recent years, the popular concept of "smart cities" has often been linked to advanced ICT use in cities, aiming at enhancing efficiency (e.g. competitiveness) and sustainability (e.g. energy saving).

The increased use of digital technologies in shaping and governing the modern and complex urban system, as a "cyber world", has led to an avalanche of 
INDEPENDENT JOURNAL OF MANAGEMENT \& PRODUCTION (IJM\&P)

http://www.ijmp.jor.br

v. 10, n. 3, May - June 2019

ISSN: 2236-269X

DOI: 10.14807/ijmp.v10i3.792

digital data systems, called "big data". Such data may relate to a variety of phenomena, such as: use of public facilities, traffic flows, emergency services, weather conditions, mobile phone usage, or crowd emergency, etc, often in a realtime context. Smart Cities use data e and make them available for scientific analysis and planning with a view to fostering the urban performance for the city organization itself, for the residents and for the business sector.

\subsection{Dimensions of Smart Cities}

According to the Global Cities Institute-GCI (2015), a city infrastructure comprises waste management, water supply, sanitation, energy, telecommunications systems and mobility networks. GCl sets, through the ISO 37120, the Global City Indicators Facility (GCIF indicator) to measure the quality, performance and resilience of services in the sustainable development of communities, which can also provide a comparison with the "smart cities" competitors.

On the other hand, many authors have been setting criteria and dimensions to identify a "Smart City", in which dimensions, techniques and tools are mixed.

Gaur et al. (2015) declare that a Smart City provides an intelligent way to manage components, such as transport, health, energy, homes and buildings, and the entire environment.

According to the website "European Smart Cities 4.0 (2015)" and corroborated by many authors as we can see in the sequence, Smart City, or Intelligent City, is the one that brings together technology, government and society to introduce the following characteristics into the city: Smart Economy, Smart Mobility, Smart Environment, Smart People, Smart Living, and Smart Governance.

Approaches on other dimensions were not analyzed in this study, considering they are somehow related to the previous ones or because they have lesser importance than others, but it will need a more detailed study in the future.

As another contribution of this article, to understand the thematic and dimensions of Smart City can contribute for better use of the I.4.0 technological tools, for faster development and better results.

\section{METHODOLOGY}


INDEPENDENT JOURNAL OF MANAGEMENT \& PRODUCTION (IJM\&P)

http://www.ijmp.jor.br

v. 10, n. 3, May - June 2019

ISSN: 2236-269X

DOI: 10.14807/ijmp.v10i3.792

This article was an exploratory study based on literature review on the technological tools of "Industry 4.0" and dimensions of a "Smart City". After, we did a cross check of concepts, in which we tried to identify citations in many papers from several authors that could corroborate that the main technological tools from I.4.0 are being used in Smart Cities development, resulting in the content explained in the sequence.

Databases as Capes Portal, Google Scholar, Science Direct, Web of Science and Scopus were used for these researches. The key words used were: "Smart Cities, Industry 4.0, Fourth Industrial Revolution", and the research was done from 2009 to 2017. An analytical framework making correlation among Smart Cities dimensions and I.4.0 technological tools was developed.

\section{RESULTS}

In Table 1, we summarize the vision and citation of some authors related to the approaches of Smart City dimensions.

We realize that the six dimensions (indicators or figures) defined by the European Smart Cities 4.0 (2015) are among the most cited in the Table 1, in which Mobility (or Transportation) was the most mentioned with 95\% of the papers, followed by Governance (or Administration, or Management) with 80\%, Environment and People (or Citizen, or Communities) both with 65\% and Living and Economy both with 55\%. Therefore, in this study we are using the top eight defined and remarked in the table, by adding the other two more cited, Energy (or Grid) with 55\% and Buildings (or Utilities) with 45\%, thus forming the eight main dimensions, which will be used to analyze their interactions with the main I.4.0 tech tools.

a) In this study we are using some expansion of the dimensions, trying to aggregate some similar understanding from other words and expressions. Thus, we have considered:

b) Smart Economy: everything related to economy, financial and business.

c) Smart Mobility: mobility control, e.g., monitoring parking availability, traffic control (ROBLEK et al., 2016); Smart Transport (Mobility): NFC payment, quality of shipment conditions, item location, storage incompatibility detection, fleet tracking, electric vehicle charging, vehicle auto-diagnosis, management 
INDEPENDENT JOURNAL OF MANAGEMENT \& PRODUCTION (IJM\&P)

http://www.ijmp.jor.br

v. 10, n. 3, May - June 2019

ISSN: 2236-269X

DOI: 10.14807/ijmp.v10i3.792

of cars, road pricing, connected militarized defense (VERMESAN; FRIESS, 2014).

d) Smart Environment: forest fire detection, air pollution, landslide and avalanche prevention, earthquake early detection, protecting wildlife, meteorological station network, marine and coastal surveillance (VERMESAN; FRIESS, 2014).

e) Smart People: A combination of Education, Lifelong learning, Ethnic plurality and Open-mindedness (EUROPEAN SMART CITIES 4.0, 2015).

f) Smart Living: Intelligent shopping applications, energy and water use, remote control appliances, weather station, smart home appliances, gas monitoring, safety monitoring, smart jewelry, community (VERMESAN; FRIESS, 2014).

Table 1: Smart Cities figures definition

\begin{tabular}{|c|c|c|c|c|c|c|c|c|c|c|c|c|c|c|c|c|c|c|c|c|c|c|c|}
\hline \multirow{2}{*}{\multicolumn{2}{|c|}{$\begin{array}{l}\text { SMART CITIES } \\
\text { FIGURES }\end{array}$}} & \multicolumn{20}{|c|}{ Papers / Authors } & \multicolumn{2}{|c|}{ Papers citations } \\
\hline & & \begin{tabular}{|c}
$2015-$ \\
European \\
Smart Cities \\
4.0
\end{tabular} & 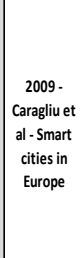 & \begin{tabular}{|c|}
$2014-$ \\
Vermesan \\
and friess- \\
liviolot \\
from \\
Research \\
and \\
Innovation \\
to Market \\
Deployment
\end{tabular} & \begin{tabular}{|c|} 
\\
2015 - Gaur \\
et al - Smart \\
City \\
Architectur \\
eand \\
Applitictions \\
based on lor \\
\end{tabular} & $\begin{array}{l}\text { 2015- Kylili } \\
\text { e Fokaides } \\
\text { The role of } \\
\text { zero energy } \\
\text { buildings }\end{array}$ & $\begin{array}{c}2016- \\
\text { ili } \\
\text { Ahmed et al } \\
\text { internet-of } \\
\text { Things- } \\
\text { Tased Smart } \\
\text { Environmen } \\
\text { ts }\end{array}$ & \begin{tabular}{|c|c}
$2016-$ \\
Anthopoulo \\
sL.- \\
Smart \\
uttopia vs \\
smart \\
reality-10 \\
smart tity \\
cases
\end{tabular} & 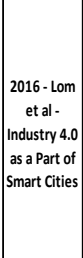 & \begin{tabular}{|c|c}
$2016-$ - jaz \\
et al- \\
Survey on \\
security \\
concerns
\end{tabular} & \begin{tabular}{|c|}
2016. \\
Maier $5 .-$ \\
Smart \\
energy \\
system for \\
smart city \\
districts
\end{tabular} & $\begin{array}{c}\text { 2016-Masek } \\
\text { et al }-A \\
\text { Harmonized } \\
\text { Perspective } \\
\text { on } \\
\text { Transportati } \\
\text { on } \\
\text { Managemen } \\
\text { tin Smart } \\
\text { Cities }\end{array}$ & \begin{tabular}{|c|} 
\\
\\
2016 Roblek \\
etal-A \\
Complex \\
View of \\
Industry 4.0 \\
\\
\end{tabular} & \begin{tabular}{|c|}
$2016-$ \\
samprif tal \\
Google \\
trends as a \\
tool for \\
smart \\
health
\end{tabular} & $\begin{array}{c}2017- \\
\text { Ahvenniemi } \\
\text { et al. } \\
\text { Differences } \\
\text { between } \\
\text { sustainable } \\
\text { and smart } \\
\text { cities }\end{array}$ & $\begin{array}{l}\text { in } \\
\text { 2017- Badii } \\
\text { et al - } \\
\text { Analysis and } \\
\text { Assessment } \\
\text { epls (IBM/ } / \\
\text { CISCO) }\end{array}$ & $\begin{array}{c}2017 \text { - Basiri } \\
\text { i et al - Smart } \\
\text { city solution } \\
\text { d } \\
\text { for } \\
t \text { sustainable } \\
\text { urban } \\
\text { developmen } \\
t\end{array}$ & 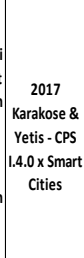 & $\begin{array}{l}2017 . \\
\text { Kummitha \& } \\
\text { Crutzen- } \\
\text { How do we } \\
\text { understand } \\
\text { smart citites }\end{array}$ & 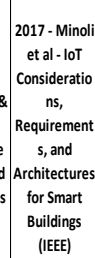 & 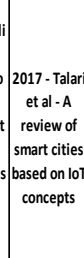 & \begin{tabular}{|c|} 
numbers \\
of \\
authors \\
citations
\end{tabular} & $\%$ \\
\hline \multirow{31}{*}{ 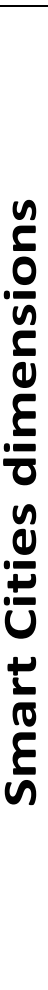 } & \begin{tabular}{|l|} 
Mobility / \\
Transportation
\end{tabular} & $x$ & $x$ & $x$ & $x$ & $x$ & $x$ & $x$ & $x$ & $x$ & $x$ & & $x$ & $x$ & $x$ & $x$ & $x$ & $x$ & $x$ & $x$ & $x$ & 19 & $95,0 \%$ \\
\hline & \begin{tabular}{|l} 
Governance / \\
Administration / \\
Management
\end{tabular} & $x$ & $x$ & $x$ & $x$ & & & $x$ & $\mathrm{x}$ & $x$ & $x$ & $x$ & $x$ & $x$ & $x$ & $x$ & $x$ & & $x$ & & $x$ & 16 & $80,0 \%$ \\
\hline & \begin{tabular}{|l|} 
Environment \\
\end{tabular} & $x$ & $x$ & $x$ & $x$ & & $x$ & & $x$ & $x$ & $x$ & & $x$ & $x$ & $x$ & & $x$ & & $x$ & & & 13 & $65,0 \%$ \\
\hline & $\begin{array}{l}\begin{array}{l}\text { People / Citizen / } \\
\text { Communities }\end{array} \\
\end{array}$ & $x$ & $x$ & $x$ & & & & $x$ & $x$ & & $x$ & & $x$ & $x$ & $x$ & $x$ & $x$ & & $x$ & & $x$ & 13 & $65,0 \%$ \\
\hline & Living & $x$ & $x$ & $x$ & & & & & $x$ & & $x$ & $x$ & $x$ & $x$ & $x$ & & $x$ & & $x$ & & & 11 & $55,0 \%$ \\
\hline & Economy & $x$ & $x$ & $\mathrm{x}$ & & & & $\mathrm{x}$ & $\mathrm{x}$ & & $\mathrm{x}$ & & $x$ & $\mathrm{x}$ & $x$ & & $x$ & & $x$ & & & 11 & $55,0 \%$ \\
\hline & Energy / Grid & & & $x$ & $x$ & $x$ & $x$ & $x$ & $x$ & $x$ & & & & & $x$ & $x$ & & $x$ & & & $x$ & 11 & $55,0 \%$ \\
\hline & Buildings / Utilities & & & $x$ & $x$ & $x$ & $x$ & & $x$ & & & & & & & & $x$ & $x$ & & $x$ & $x$ & 9 & $45,0 \%$ \\
\hline & $\begin{array}{l}\text { Industry (factory / } \\
\text { Production) }\end{array}$ & & & $x$ & $x$ & & $x$ & & & & & $x$ & $x$ & & & & & $x$ & & & & 6 & $30,0 \%$ \\
\hline & Heath & & & $x$ & $x$ & & $x$ & & & & & & & & & $x$ & & & & & $x$ & 5 & $25,0 \%$ \\
\hline & Technologies & & & & & & & $x$ & & & & $x$ & $x$ & & $x$ & & & & & & $x$ & 5 & $25,0 \%$ \\
\hline & 3 Products/Things & & & & & & & & $\mathrm{x}$ & & & & $x$ & & & $x$ & & $x$ & & & & 4 & $20,0 \%$ \\
\hline & \begin{tabular}{|l} 
Infraestructure \\
\end{tabular} & & & & & & & $x$ & & & & & $x$ & & & & $x$ & & & & $x$ & 4 & $20,0 \%$ \\
\hline & Services & & & & & & & $x$ & $x$ & & & $x$ & & & & $x$ & & & & & & 4 & $20,0 \%$ \\
\hline & Security / Safety & & & & $x$ & & & & & & & & & & & $\mathrm{x}$ & & & & & $x$ & 3 & $15,0 \%$ \\
\hline & Communication & & & $\mathrm{x}$ & & & & & & $x$ & & & & & & $x$ & & & & & & 3 & $15,0 \%$ \\
\hline & Devices & & & & & & & & $x$ & & & & $x$ & & & & & & & & & 2 & $10,0 \%$ \\
\hline & Society & & & & & & & & & & & & & & $x$ & & & $x$ & & & & 2 & $10,0 \%$ \\
\hline & - Retail / commerce & & & & & & & & & $x$ & & & & & & & & $x$ & & & & 2 & $10,0 \%$ \\
\hline & Meters & & & & & & $x$ & & $x$ & & & & & & & & & & & & & $\frac{2}{2}$ & $\begin{array}{l}10,0,10 \% \\
\end{array}$ \\
\hline & Eduaction & & & & & & & & & & & & & & & $x$ & & & & & $x$ & 2 & $10,0 \%$ \\
\hline & Equipments & & & & & & & & & & & & $x$ & & & & & & & & & 1 & $5,0 \%$ \\
\hline & Project & & & & & & & & & & & & $\mathrm{x}$ & & & & & & & & & 1 & $5,0 \%$ \\
\hline & Homes & & & & & & $x$ & & & & & & & & & & & & & & & 1 & $5,0 \%$ \\
\hline & \begin{tabular}{|l|} 
Lighting \\
\end{tabular} & & & & & & & $x$ & & & & & & & & & & & & & & 1 & $5,0 \%$ \\
\hline & Things / Objects & & & & & & $x$ & & & & & & & & & & & & & & & 1 & $5,0 \%$ \\
\hline & \begin{tabular}{|l|} 
Logistic \\
\end{tabular} & & & & & & & & $x$ & & & & & & & & & & & & & 1 & $5,0 \%$ \\
\hline & \begin{tabular}{|l|} 
Appliances \\
\end{tabular} & & & & & & & & $x$ & & & & & & & & & & & & & 1 & $5,0 \%$ \\
\hline & \begin{tabular}{|l|} 
Business \\
\end{tabular} & & & & & & & & & & & & & & & $x$ & & & & & & 1 & $5,0 \%$ \\
\hline & \begin{tabular}{|l|} 
water \\
\end{tabular} & & & & & & & & & & & & & & & $\mathrm{x}$ & & & & & & 1 & $5,0 \%$ \\
\hline & Parking & & & & & & & $x$ & & & & & & & & & & & & & & 1 & $5,0 \%$ \\
\hline
\end{tabular}

Source: Storolli, Makiya and Cesar, 2017 
INDEPENDENT JOURNAL OF MANAGEMENT \& PRODUCTION (IJM\&P)

http://www.ijmp.jor.br

v. 10, n. 3, May - June 2019

ISSN: 2236-269X

DOI: 10.14807/ijmp.v10i3.792

a) Smart Buildings: Smart infrastructure when smart devices are incorporated into buildings (ROBLEK et al., 2016). Perimeter access control, liquid presence, indoor climate control, intelligent thermostat, intelligent fire alarm, intrusion detection systems, motion detection, preservation of art and goods, residential irrigation (VERMESAN; FRIESS, 2014).

b) Smart Grids: or Smart Energy, photovoltaic installations, wind turbines, water flow, radiation levels, power supply controllers (VERMESAN; FRIESS, 2014).

In Table 2, we made a correlation between the Smart Cities dimensions figures top eight defined in Table 1 and the main I.4.0 technical tools, to understand their relations and how they are connected, once we are supposing technology is the main booster for I.4.0 and Smart Cities as well.

The numbers in the matrix correlation in Table 2 (1 up to 41) are related to the statements made by the authors described below, which are the motivation of this study:

a) Kummitha and Crutzen (2017): Information and Communication Technologies (ICTs), the proponents of such a technology-centered approach go on to elaborate the implications of 'smart' technology in other contexts of city life, such as 'smart mobility', 'smart environment', 'smart people', 'smart economy', 'smart living', and 'smart governance'.

b) Kummitha and Crutzen (2017): Information and Communication Technologies (ICTs) are claimed to be at the core of the smart city discourse (GRAHAM; MARVIN, 2001), which emphasizes "enhancing the socio economic, ecological, logistic and competitive performance of cities" (KOURTIT; NIJKAMP, 2012 p.93).

c) Kummitha and Crutzen (2017): the intent of the smart city is to offer its citizens the highest possible quality of urban life solutions (BAKICl et al., 2013; COCCHIA, 2014). The adoption of technologies, mainly 'internet of things' (IOT) and their integration into the city infrastructure, to advance effectiveness and efficiency in the city environment. 
INDEPENDENT JOURNAL OF MANAGEMENT \& PRODUCTION (IJM\&P)

http://www.ijmp.jor.br

v. 10, n. 3, May - June 2019

ISSN: 2236-269X

DOI: 10.14807/ijmp.v10i3.792

Table 2: Correlation between Smart Cities dimensions and I.4.0 technological tools

\begin{tabular}{|c|c|c|c|c|c|c|c|c|c|}
\hline \multirow{2}{*}{\multicolumn{2}{|c|}{ Industry 4.0}} & \multicolumn{8}{|c|}{ Smart Cities dimensions - European Smart Cities 4.0 (2015) } \\
\hline & & \multirow[t]{2}{*}{$\begin{array}{l}\text { 1. Smart } \\
\text { Economy }\end{array}$} & \multirow{2}{*}{$\begin{array}{c}\begin{array}{c}\text { 2. Smart } \\
\text { Mobility }\end{array} \\
41\end{array}$} & \multirow{2}{*}{\begin{tabular}{|c|}
$\begin{array}{c}\text { 3. Smart } \\
\text { Environme } \\
\text { nt }\end{array}$ \\
41 \\
\end{tabular}} & \multirow[t]{2}{*}{$\begin{array}{l}\text { 4. Smart } \\
\text { People }\end{array}$} & \multirow[t]{2}{*}{$\begin{array}{l}\text { 5. Smart } \\
\text { Living }\end{array}$} & \multirow[t]{2}{*}{$\begin{array}{c}\text { 6. Smart } \\
\text { Governance }\end{array}$} & \multirow[t]{2}{*}{$\begin{array}{l}\text { 7. Smart } \\
\text { Buildings }\end{array}$} & \multirow{2}{*}{$\begin{array}{c}\text { 8. Smart } \\
\text { Grids } \\
41\end{array}$} \\
\hline \multirow{21}{*}{ 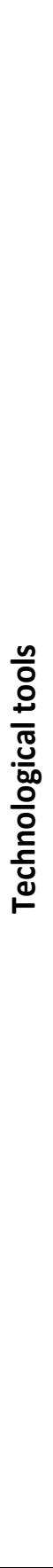 } & $\begin{array}{l}\text { Additive Manufacturing } \\
\text { (3D print) }\end{array}$ & & & & & & & & \\
\hline & $\begin{array}{l}\text { AGV (Automatic Guided } \\
\text { Vehicle) }\end{array}$ & 25 & & & & 25 & & & \\
\hline & \begin{tabular}{|l|} 
Application computer \\
programs (APPS - Waze, \\
Uber, etc)
\end{tabular} & $4,13,34$ & $4,14,34$ & 4 & 4,34 & 4 & $4,13,34$ & & 18,34 \\
\hline & $\begin{array}{l}\text { Artificial Intelligence } \\
\text { (AI) }\end{array}$ & 24 & & & & 24 & & & \\
\hline & Automation System & 22 & 22 & 4,22 & & 22 & 22 & 4 & \\
\hline & Big Data Analytics & $\begin{array}{c}4,13,22 \\
23,24,33 \\
39,40\end{array}$ & $\begin{array}{c}4,19,21 \\
22,23,33 \\
39,40 \\
\end{array}$ & $4,22,23,40$ & $4,33,39,40$ & $\begin{array}{c}4,11,22 \\
23,24,39 \\
40\end{array}$ & $\begin{array}{c}4,13,22 \\
23,33,39 \\
40\end{array}$ & 4,23 & $\begin{array}{l}4,15,19 \\
21,23,33 \\
39\end{array}$ \\
\hline & Big Data Warehouse & $\begin{array}{c}4,13,22 \\
23,24,33 \\
39,40\end{array}$ & $\begin{array}{c}14,19,21 \\
22,23,33 \\
39,40 \\
\end{array}$ & $4,22,23,40$ & $4,33,39,40$ & $\begin{array}{c}4,11,22 \\
23,24,39 \\
40\end{array}$ & $\begin{array}{c}4,13,22 \\
23,33,39 \\
40\end{array}$ & 4 & $\begin{array}{l}4,15,19 \\
21,23,33 \\
39\end{array}$ \\
\hline & Cloud Computing & $\begin{array}{c}10,13,22 \\
23,24,33 \\
39,40\end{array}$ & $\begin{array}{c}14,19,21 \\
22,23,33 \\
39,40\end{array}$ & $\begin{array}{c}10,22,23 \\
37,40\end{array}$ & $\begin{array}{c}33,37,39 \\
40\end{array}$ & $\begin{array}{c}10,22,23 \\
24,37,39 \\
40\end{array}$ & $\begin{array}{c}13,22,23 \\
33,37,39 \\
40\end{array}$ & 23,26 & $\begin{array}{c}15,19,21, \\
23,26,33, \\
39\end{array}$ \\
\hline & $\begin{array}{l}\text { CPS (Cyber Physical } \\
\text { System) }\end{array}$ & $24,25,35$ & 35 & & 35 & 24,25 & 35 & & 35 \\
\hline & Cyber Security & $\begin{array}{l}12,22,23 \\
33,39,40\end{array}$ & $\begin{array}{l}12,14,16 \\
19,22,23 \\
33,39,40\end{array}$ & $\begin{array}{l}12,16,22 \\
23,40\end{array}$ & $\begin{array}{c}16,33,39 \\
40\end{array}$ & $\begin{array}{l}12,16,22 \\
23,39,40\end{array}$ & $\begin{array}{l}12,16,22 \\
23,33,39 \\
40\end{array}$ & 16,23 & $\begin{array}{c}15,19,23 \\
33,39\end{array}$ \\
\hline & Data Mining / BI & $\begin{array}{c}4,13,22 \\
23,24,33 \\
39,40 \\
\end{array}$ & $\begin{array}{c}14,19,21 \\
22,23,33 \\
39,40 \\
\end{array}$ & $4,22,23,40$ & $4,33,39,40$ & $\begin{array}{c}4,11,22 \\
23,24,39 \\
40\end{array}$ & $\begin{array}{c}4,13,22 \\
23,33,39 \\
40\end{array}$ & 4 & $\begin{array}{l}4,15,19 \\
21,23,33 \\
39\end{array}$ \\
\hline & Horizontal Integration & 22 & 22 & 22 & & 22 & 22 & & \\
\hline & IoD (Internet of Data) & $\begin{array}{l}13,20,22, \\
23,24,30 \\
33,39,40\end{array}$ & $\begin{array}{c}14,16,19 \\
21,22,23 \\
30,31,33 \\
36,38,39 \\
40\end{array}$ & $\begin{array}{c}2,3,16,22 \\
23,30,36 \\
37,38,40\end{array}$ & $\begin{array}{c}16,33,37 \\
39,40\end{array}$ & $\begin{array}{c}3,16,20 \\
22,23,24 \\
30,37,38 \\
39,40\end{array}$ & $\begin{array}{l}13,16,20 \\
22,23,33 \\
37,39,40\end{array}$ & $\begin{array}{c}16,23,26 \\
36,38\end{array}$ & $\begin{array}{c}15,18,19, \\
20,21,23, \\
26,31,32, \\
33,36,38, \\
39\end{array}$ \\
\hline & IoS (Internet of Service) & $\begin{array}{l}13,20,22, \\
23,24,30 \\
33,39,40\end{array}$ & $\begin{array}{c}14,16,19 \\
21,22,23 \\
30,31,33 \\
36,38,39 \\
40\end{array}$ & $\begin{array}{l}2,3,16,22 \\
23,30,36 \\
37,38,40\end{array}$ & $\begin{array}{c}16,33,37 \\
39,40\end{array}$ & $\begin{array}{c}16,20,23 \\
24,30,37 \\
38,39,40\end{array}$ & $\begin{array}{l}13,16,20 \\
22,23,33 \\
37,39,40\end{array}$ & $\begin{array}{c}16,23,26 \\
36,38\end{array}$ & $\begin{array}{c}15,18,19, \\
20,21,23, \\
26,31,32, \\
33,36,38, \\
39\end{array}$ \\
\hline & IoT (Internet of Things) & $\begin{array}{c}1,10,13 \\
20,22,23 \\
24,28,29 \\
30,33,39 \\
40\end{array}$ & $\begin{array}{c}1,8,14,16 \\
19,21,22 \\
23,27,28 \\
30,31,33 \\
36,38,39 \\
40\end{array}$ & $\begin{array}{c}1,2,3,4,7 \\
8,16,22 \\
23,27,28 \\
30,36,37 \\
38,40\end{array}$ & $\begin{array}{c}1,16,27 \\
33,37,39 \\
40\end{array}$ & $\begin{array}{c}1,7,9,16 \\
20,22,23, \\
24,27,28 \\
30,37,38 \\
39,40\end{array}$ & $\begin{array}{l}1,13,16 \\
20,22,23 \\
27,28,33 \\
37,39,40\end{array}$ & $\begin{array}{l}8,16,23 \\
26,36,38\end{array}$ & $\begin{array}{c}15,18,19, \\
20,21,23, \\
26,28,31, \\
32,33,36, \\
38,39\end{array}$ \\
\hline & Simulation Software & & & & & & & 26 & 26 \\
\hline & $\begin{array}{l}\text { Smart devices (phone, } \\
\text { tablet, watch) }\end{array}$ & $\begin{array}{l}5,10,13 \\
24,30,39\end{array}$ & $\begin{array}{c}5,14,16 \\
21,30,31 \\
39\end{array}$ & $\begin{array}{c}5,10,16 \\
30,37\end{array}$ & $5,16,37,39$ & $\begin{array}{c}5,10,16 \\
24,30,37 \\
39\end{array}$ & $\begin{array}{c}5,13,16 \\
37,39\end{array}$ & 5,16 & $\begin{array}{c}18,21,31 \\
32,39\end{array}$ \\
\hline & Smart Factory & 24 & & & & 24 & & & \\
\hline & $\begin{array}{l}\text { Smart Identification } \\
\text { (RFID tag / NFC / } \\
\text { Bluetooth) }\end{array}$ & 17 & 14,17 & 17 & & & & & \\
\hline & Smart Sensing & $\begin{array}{c}4,10,13 \\
23,39\end{array}$ & $4,21,23,39$ & $4,10,23,37$ & 37,39 & $\begin{array}{c}4,10,23 \\
37,39 \\
\end{array}$ & $\begin{array}{c}4,13,23 \\
37,39 \\
\end{array}$ & $4,23,26$ & $\begin{array}{l}4,18,21 \\
23,26,39\end{array}$ \\
\hline & Traceability & & 14 & & & & & & 15 \\
\hline
\end{tabular}

Source: Storolli, Makiya and Cesar, 2017

a) Ljaz et al. (2016): The smart city is designed, constructed, and maintained by using highly advanced integrated technologies, which include sensors, electronics, and networks that are linked with computerized systems comprised of databases, tracking, and decision-making algorithms. 
b) Ljaz et al. (2016): The term global village seems very coherent with the smart city, as urbanization is dependent on the latest technologies and Internet. The concept is also influenced by the industries promoting and selling their products such as GPS, iPad, smartphones and other technologies. Hence the smart city promises smarter growth. It is said that proper investments in developing the systems of a city through embedded technologies will help to promote an immense growth in the economic system as well. Certain pioneering cities that are considered the next generation smart cities.

c) Ljaz et al. (2016): It should be considered that the latest information and communication technologies (ICT) that are the core part of an efficient smart city are the Internet of things (IOT), smartphone technology, RFID (Radio Frequency Identification System), smart meters, semantic web, linked data, ontologies, artificial intelligence, cloud computing, collective intelligence, software, smart apps, and biometrics.

d) Ljaz et al. (2016): The concept of IoT plays a vital role in the development of an ideal and secure smart city.

e) Ljaz et al. (2016): The loT is considered as a major research and innovation idea that leads to a lot of opportunities for new services by interconnecting physical and virtual worlds with a huge amount of electronics distributed in different places, including houses, vehicles, streets, buildings and many other environments.

f) Ljaz et al. (2016): The concept of Smart Cities is distinguished on the fact that it is solely dependent on embedded systems, smart technologies and the IoT. In general term, a Smart City relies on information technology and on the embedded infrastructure to facilitate it for a better living standard.

g) Ljaz et al. (2016): The issues of information security also need to be addressed for a better economic development of a Smart City. The requirements of ideally secured and reliable smart cities need to be recognized considering most of the technologies, specially focusing on IoT, cloud computing, real world user interfaces, smart sensors, smartphones, semantic web etc (linked to the item 7). 
h) Ljaz et al. (2016): A very important factor that plays a key role in developing a Smart City is "big data". The production of large data sets in a smart city is an inevitable phenomenon including national consensuses, government records, and other information about the citizens. From such data, the smart cities can extract very important information, helping real time analysis and ubiquitous computing. The author elaborates that though the big data provides various opportunities for smarter life, it still brings challenges of security and privacy.

i) Ljaz et al. (2016): the information security in Smart City is mostly dependent on three factors: governance, socioeconomic and technological factors. These factors influence and identify the information security issues in a smart city. The ICT technologies work together to form a smart city, but, although they implement the whole infrastructure of a smart city and provide solutions for information security problems, they also trigger new concerns and problems regarding security, privacy, protection and resilience.

j) Ljaz et al. (2016): "...the governance and socioeconomic factors are dependent on the technological factors as these are implemented in a smart city through technology".

k) Ljaz et al. (2016): Smart mobility may cause privacy concerns as personal information disclosure could happen when collecting, publishing, and utilizing trace data. Here, localization techniques include GPS, GSM, WiFi, Bluetooth, and RFID because centric servers do not need to know device IDs. Some of the smartphone apps that provide services of smart mobility take mobile data and use trace analysis and data mining techniques.

I) Ljaz et al. (2016): Energy and utility services are increasingly relying on smart grids that use bidirectional communication with the users in order to manage the distributed energy efficiently. Cloud computing also plays its role by providing features that are well suited for smart grid software platforms. Data security and privacy remain top concerns for utilities and users, which is playing a crucial setback in the adoption of smart grids.

m) Ljaz et al. (2016): The Internet of Things (IoT) incorporates a huge number of distinguished and heterogeneous devices and gives free access to information for various online services for smart cities. IOT plays a gigantic role 
in developing and maintaining the services of a smart city, hence making the issue of secure information flow a huge task with respect to it.

n) Ljaz et al. (2016): Radio Frequency Identification (RFID) tags are being used immensely in the various components of smart city including smart environment, industry and mobility, etc. It has brought significant benefits in many other areas as well through improving real-time information visibility and traceability.

o) Ljaz et al. (2016): Smart grids play a core part in a smart city regarding energy deployment and management. These are actually communicating instruments including sensors and communication networks that help in communicating data in real time.

p) Ahvenniemi et al. (2017): A common understanding, also shared by the European Commission (2012), is that diverse technologies help in achieving sustainability in smart cities. According to the latter source, smart cities and communities focus on the intersection between energy, transport and ICT, which are also the fields that have received most of the EU's public smart cities related funding (under the Horizon 2020 program "smart cities and communities").

q) Ahvenniemi et al. (2017): Caragliu et al. (2011) state that a city is smart "when investments in human and social capital and traditional (transport) and modern (ICT) communication infrastructure fuel sustainable economic growth and a high quality of life, with a wise management of natural resources, through participatory governance".

r) Badii et al. (2017): In general, all smart city solutions must cope with big data volume, variety, and veracity. Open data as static data are not the main source of information in the city. Most of the big data problems connected to the smart city platform are related to real-time data as the vehicle and human mobility in the city, energy consumption, health care, and IOT. It is obvious to state that, cloud and distributed systems approaches are at the basis of the big data solutions provided for smart city, as well as IOT solutions at the basis for collecting data from sensors and devices in the city. 
s) Basiri et al. (2017): Smart City Characteristics: In order to enable this to happen, a number of key characteristics are required:

- The city will be instrumented to allow the collection of increasing amounts of data about city life;

- The data from different sources and city systems will be available to be easily aggregated together to gain far greater insight into what is going on in the city;

- In addition, analytics and decision-making systems will be used, so that this knowledge can be used effectively, both by city managers and planners, and by the citizen, to support real time decision making and enable effective actions to be identified that will enable future requirements to be met;

- The city will also be automated, to enable appropriate city functions to be delivered reliably, and effectively, without the need of direct human intervention;

- The city will have a network of collaborative spaces, to enable dynamic communities that will spur innovation and growth and enhance citizen well-being; and

- The continual interaction between the physical and digital worlds enables the decision-making processes to be much more open and inclusive, so that citizens, policy makers and businesses can work together effectively to manage the life of the city for the benefit of all (ISO, 2014).

t) Basiri et al. (2017): Benefits of Smart City - The application of information technology in Smart Cities can produce various benefits:

- Reducing resource consumption, notably energy and water; hence contributing to reductions in CO2 emissions (NYC, 2007);

- Making new services available to citizens and commuters, such as realtime guidance on how best to exploit multiple transportation modalities; 
- Revealing how demands for energy, water and transportation peak at a city scale so that city managers can collaborate to smooth these peaks and to improve resilience.

The widespread use of digital sensors and digital control systems for the control and operation of urban infrastructure, including traffic sensors, building management systems, digital utility meters, and so forth, have become feasible as a result of recent progress in technology.

u) Karakose and Yetis (2017): Smart City aims to achieve strong city characteristics such as economy and culture, with more efficient use of the physical infrastructure by using cyber computations such as artificial intelligence and data analytics. On the other hand, electronic platforms, which are also used for making people more active in choices about city and even country, are utilized in smart city concept. Using such platforms not only make people's life easier but also provide us data to analyze other components of smart city. So, we use Internet and mobile platforms for taking orders. With the electronically gathered information, some estimates can be made to make the production process more efficient (CPS). Furthermore, the information can be used for learning algorithms, and self-modified systems can be achieved.

v) Karakose and Yetis (2017): Producing mass-customized products is more complex than producing regular ones. In order to find the optimal solutions, application software and hardware should work in harmony. Such a system depends on similar characteristics with CPS. Machines in a factory work according to pipeline method. So, when a machine works on one product, another machine works on probably another one. This makes the system responsible for managing all machines simultaneously one by one. So, the production can be shipped easily by the autonomy shipping vehicles that are a part of smart city.

w) Minoli et al. (2017): The large footprint defines a sizable market opportunity for technical solutions incorporated in Building Management Systems (BMSs), which are now increasingly based on loT principles. Inexpensive sensors are emerging, and user-friendly applications are becoming available, often as a software of cloud-provided service; these developments are now driving the 
INDEPENDENT JOURNAL OF MANAGEMENT \& PRODUCTION (IJM\&P)

http://www.ijmp.jor.br

v. 10, n. 3, May - June 2019

ISSN: 2236-269X

DOI: 10.14807/ijmp.v10i3.792

deployment of the IoT in building applications. BMS is a comprehensive platform that is employed to monitor and control a building's mechanical and electrical equipment; they are used to manage loads and enhance efficiency, thus having the ability to reduce the energy needed to illuminate, heat, cool and ventilate a building.

x) Talari et al. (2017): There have been a lot of service domain applications that utilize an IOT substructure to simplify operations in air and noise pollution control, the movement of cars, as well as surveillance and supervision systems. The developments on the Internet provide a substructure that enables a lot of persons to interlink with each other.

y) Talari et al. (2017): Providing loT improves cities and affects the different features of humans' life by creating cost-effective municipal services, enhancing public transformation, reducing traffic congestion, keeping citizens safe and healthier. Moreover, it plays a vital role in the national level associated with policy making (e.g., energy conservation and pollution reduction), monitoring systems, and needed infrastructures. Thus, it helps to supply a system with more efficiency, lower cost and more secure operation through energy conservation rules, economic attention as well as reliability level.

z) Talari et al. (2017): According to the US National Intelligence Council, the IoT is one of the most efficient sources of US economic profits on the way to 2025.

aa)Roblek et al. (2016): the "smartness economy" will change the way of creating added value. Sources of production may change, but additional services will be accessible via Internet. This can already be seen in smart mobility, automobile leasing, and various examples relating to the industrial Internet, mechanics, and heavy industry; in cases of smart homes (smart devices), which, for example, may include, along with a TV, a fridge and a game console equipped with an IP number and connected to the IOT (MARTIN, 2015). These days the Internet relates to more than a billion people through personal computers, tablets, and smartphones. 
bb)Lom et al. (2016): In the concept of Industry 4.0, the Internet of Things (IOT) shall be used for the development of so-called smart products. Important aspects of the I.4.0 are Internet of Services (IOS), which includes especially intelligent transport and logistics (smart mobility, smart logistics), as well as Internet of Energy (loE), which determines how the natural resources are used in proper way (electricity, water, oil, etc.).

cc) Lom et al. (2016): The loT concept is expected to offer advanced connectivity of devices and products. Each device connecting to internet is being expecting having a set of smart services called Internet of Services (IoS). The interconnection of these embedded devices is expected to usher in automation in nearly all fields, while also enabling advanced applications such as a smart grid.

dd)Anthopoulos (2016): The Smart Sustainable City is an innovative city that uses information and communication technologies (ICT) and other means to improve quality of life, efficiency of urban operation and services, and competitiveness, while ensuring that it meets the needs of present and future generations, with respect to economic, social and environmental aspects (Mobility, People, Governance, Economy, Grid).

ee)Anthopoulos (2016): For a Smart City, visitor gains come up from the city's performance in terms of planning (urban spaces and parks, streets, sidewalks etc.) and utilities' efficiency (transportation networks, bike and car sharing etc.), and then by the accompanied intelligence (creativity, Wi-Fi and App identification, smart stations etc.).

ff) Anthopoulos (2016): A brief view to the Future Smart City according to the examined cases' visions can be depicted, which shows cities try to enhance their living - the latest by 2050 - with cyber-physical integration on existing, renovated and new districts; with sustainably planned new districts; with digital economy's growth; and with city monitoring.

gg)Ahmed et al. (2016): The forecast of such significant growth shows IoT will become the fabric of modern societies to realize the vision of smart environments. The integration of IoT with a smart environment extends the capabilities of smart objects by enabling the user to monitor the environment 
INDEPENDENT JOURNAL OF MANAGEMENT \& PRODUCTION (IJM\&P)

http://www.ijmp.jor.br

v. 10, n. 3, May - June 2019

ISSN: 2236-269X

DOI: 10.14807/ijmp.v10i3.792

from remote sites. The work on loT-based smart environments can generally be classified into the following areas: smart cities, smart homes, smart grid, smart buildings, smart transportation, smart health, and smart industry.

hh) Gaur et al. (2015): The main aim is to connect all sorts of things (sensors and IoT's) that can help in making the life of citizens more comfortable and safer. An example is provided by communication services in the home domain for connecting telephone devices and PC through the internet. In the case of the Government sector, cloud and communication services are combined to obtain a better governance system. In the case of the health sector, communication technologies can be used to connect health statistics, medication and location of the patient from a remote location, thus helping to achieve a Smart Health system. Hence, with Smart City and communication technologies we can provide a more secure and convenient infrastructure for better living in the Smart City environment.

ii) Vermesan and Friess (2014): The Internet of Things and Services makes possible to create networks incorporating the entire manufacturing process that convert factories into a smart environment. The IoT application domains identified by IERC (Internet of Things European Research Cluster) are based on inputs from experts, surveys and reports. The IOT application covers "smart" environment/spaces and self-aware things devices in domains such as smart transportation, buildings, city, lifestyle, retail, agriculture, factory, supply chains, emergency, health, living, care, user interaction, culture and tourism, environment, food, energy-grid, mobility, digital society and health applications.

jj) Vermesan and Friess (2014): The deployment of ICT to create "smart cities" is gaining momentum in Europe, accentuated by the numerous pilot projects running at regional, country and EU levels. Initiatives revolve around energy and water efficiency, mobility, infrastructure and platforms for open cities, citizen involvement, and public administration services. Projects are carried out in the form of collaborative networks established between the research community, business (economy), the public sector, citizens and the wider community, and they foster an open innovation approach. Technologies such as smart metering, wireless sensor networks, open platforms, high-speed 
INDEPENDENT JOURNAL OF MANAGEMENT \& PRODUCTION (IJM\&P)

http://www.ijmp.jor.br

v. 10, n. 3, May - June 2019

ISSN: 2236-269X

DOI: 10.14807/ijmp.v10i3.792

broadband and cloud computing are key building blocks of the smart city infrastructure.

kk) Caragliu et al. (2009): A recent and interesting project conducted by the Centre of Regional Science at the Vienna University of Technology identifies six main 'axes' (dimensions) along which a ranking of 70 European middle size cities can be made. These axes are: a smart economy; smart mobility; a smart environment; smart people; smart living; and, finally, smart governance. These six axes connect with traditional regional and neoclassical theories of urban growth and development, focused on regional competitiveness, transport and ICT economics, natural resources, human and social capital, quality of life, and participation of societies in cities.

II) Gerlitz (2015): From the environmental responsibility perspective, design integration and implementation in the industry 4.0 context enables organizations to introduce more environmentally friendly practices. New integrated design approaches combined with key innovation creating technologies (3D printing) have positive impact on corporation strategic orientation: from the logistical point of view, production of spare parts on demand reduces logistical practices, and thus, environmental impact. Energy and fuel consumption can be efficiently saved through reduced logistical interactions, as the need for warehouses and their integration in the supply chains become unnecessary. As a result, the environmental impact is also reduced through saved energy usage and fuels used to transport and distribute spare parts concerned. This, in turns, allows greater sustainability of product / service through material savings, reduced resource usage and ecological mindset.

\section{DISCUSSION}

We recognize Smart Cities as an innovation ecosystem for the society, towards deeper understanding of challenges and demands related to citizen's quality of life reconfiguration, based on new solutions in infrastructure and services. On the other hand, the fourth Industrial Revolution drives the implementations using many of the techniques and technological tools developed within the scope of the term industry 4.0 as a basis. 
INDEPENDENT JOURNAL OF MANAGEMENT \& PRODUCTION (IJM\&P)

http://www.ijmp.jor.br

v. 10, n. 3, May - June 2019

ISSN: 2236-269X

DOI: 10.14807/ijmp.v10i3.792

Seen in these terms, Table 3 below shows the main relationships among Smart Cities Dimensions and Technological Tools of Industry 4.0 and, especially, the main relationships identified among them by the research carried out.

Table 3: Number of citations in the Correlation Smart Cities dimensions and I.4.0 technological tools

\begin{tabular}{|c|c|c|c|c|c|c|c|c|c|c|}
\hline \multirow{2}{*}{\multicolumn{2}{|c|}{ Industry 4.0}} & \multicolumn{8}{|c|}{ Smart Cities dimensions - European Smart Cities 4.0 (2015) } & \multirow[b]{2}{*}{$\begin{array}{c}\text { total } \\
\text { citation }\end{array}$} \\
\hline & & $\begin{array}{l}\text { 1. Smart } \\
\text { Economy }\end{array}$ & $\begin{array}{l}\text { 2. Smart } \\
\text { Mobility }\end{array}$ & $\begin{array}{c}\text { 3. Smart } \\
\text { Environment }\end{array}$ & $\begin{array}{l}\text { 4. Smart } \\
\text { People }\end{array}$ & $\begin{array}{l}\text { 5. Smart } \\
\text { Living }\end{array}$ & $\begin{array}{c}\text { 6. Smart } \\
\text { Governance }\end{array}$ & $\begin{array}{l}\text { 7. Smart } \\
\text { Buildings }\end{array}$ & $\begin{array}{l}\text { 8. Smart } \\
\text { Grids }\end{array}$ & \\
\hline \multirow{21}{*}{ 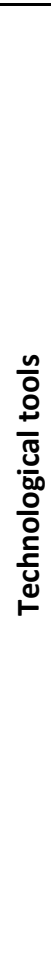 } & $\begin{array}{l}\text { Additive Manufacturing } \\
\text { (3D print) }\end{array}$ & & 1 & 1 & & & & & 1 & 3 \\
\hline & $\begin{array}{l}\text { AGV (Automatic Guided } \\
\text { Vehicle) }\end{array}$ & 1 & & & & 1 & & & & 2 \\
\hline & $\begin{array}{l}\text { Application computer } \\
\text { programs (APPS - Waze, } \\
\text { Uber, etc) }\end{array}$ & 3 & 3 & 1 & 2 & 1 & 3 & & 2 & 15 \\
\hline & Artificial Intelligence (AI) & 1 & & & & 1 & & & & 2 \\
\hline & Automation System & 1 & 1 & 2 & & 1 & 1 & 1 & & 7 \\
\hline & Big Data Analytics & 8 & 8 & 4 & 4 & 7 & 7 & 2 & 7 & 47 \\
\hline & Big Data Warehouse & 8 & 8 & 4 & 4 & 7 & 7 & 1 & 7 & 46 \\
\hline & Cloud Computing & 8 & 8 & 5 & 4 & 7 & 7 & 2 & 7 & 48 \\
\hline & $\begin{array}{l}\text { CPS (Cyber Physical } \\
\text { System) }\end{array}$ & 3 & 1 & & 1 & 2 & 1 & & 1 & 9 \\
\hline & Cyber Security & 6 & 9 & 5 & 4 & 7 & 7 & 2 & 5 & 45 \\
\hline & Data Mining / BI & 8 & 8 & 4 & 4 & 7 & 7 & 1 & 7 & 46 \\
\hline & Horizontal Integration & 1 & 1 & 1 & & 1 & 1 & & & 5 \\
\hline & IoD (Internet of Data) & 9 & 13 & 10 & 5 & 11 & 9 & 5 & 13 & 75 \\
\hline & IoS (Internet of Service) & 9 & 13 & 10 & 5 & 9 & 9 & 5 & 13 & 73 \\
\hline & IoT (Internet of Things) & 13 & 17 & 16 & 7 & 15 & 12 & 6 & 14 & 100 \\
\hline & Simulation Software & & & & & & & 1 & 1 & 2 \\
\hline & $\begin{array}{l}\text { Smart devices (phone, } \\
\text { tablet, watch) }\end{array}$ & 6 & 7 & 5 & 4 & 7 & 5 & 2 & 5 & 41 \\
\hline & Smart Factory & 1 & & & & 1 & & & & 2 \\
\hline & $\begin{array}{l}\text { Smart Identification } \\
\text { (RFID tag / NFC / } \\
\text { Bluetooth) } \\
\end{array}$ & 1 & 2 & 1 & & & & & & 4 \\
\hline & Smart Sensing & 5 & 4 & 4 & 2 & 5 & 5 & 3 & 6 & 34 \\
\hline & Traceability & & 1 & & & & & & 1 & 2 \\
\hline
\end{tabular}

Source: Storolli, Makiya and Cesar, 2017

We found specific stronger relations between the IoT \& Smart Mobility (17 citations), IoT \& Smart Environment (16), IoT \& Smart Living (15), IOT \& Smart Grids (14), IoT \& Smart Economy (13), IoS \& Smart Mobility (13), IoS \& Smart Grids (13), IoD \& Smart Mobility (13), IoD \& Smart Grids (13) and IoT \& Smart Governance (12). IoT has strong relation with 6 of 8 Smart Cities dimensions in the top 10 and a little bit lower in relation with People and Building. It was identified 100 citations of IoT with all Smart Cities dimensions as represented in the last column of the table.

In the sequence, IOD and loS have strong relations to the Smart Mobility and Grids dimensions, and good relations with Smart Economy, Environment, Living and Governance, getting 75 and 73 citations, respectively. Therefore, we must consider both strong relationship with IoT and their intrinsic properties of operationalization. 
INDEPENDENT JOURNAL OF MANAGEMENT \& PRODUCTION (IJM\&P)

http://www.ijmp.jor.br

v. 10, n. 3, May - June 2019

ISSN: 2236-269X

DOI: 10.14807/ijmp.v10i3.792

Graphic 1 represents the Pareto analysis as a technique to identify the most used tools in the studied dimensions, to reconstruct the environment in the most connected and intelligent cities. So, we have also identified the technological tools as Big Data Warehouse \& Analytics, Cloud Computing, Cyber Security, Data Mining, BI and Smart Devices, which have been pushing the development of cities and improving the quality of life, citizens security and energy efficiency. Despite of not being among the top, smart devices can also be considered, because most of the "tech tools" mentioned use them as interface with human beings.

Graphic 1: Pareto of top 10 levels citations in the Correlation Smart Cities dimensions and I.4.0 technological tools

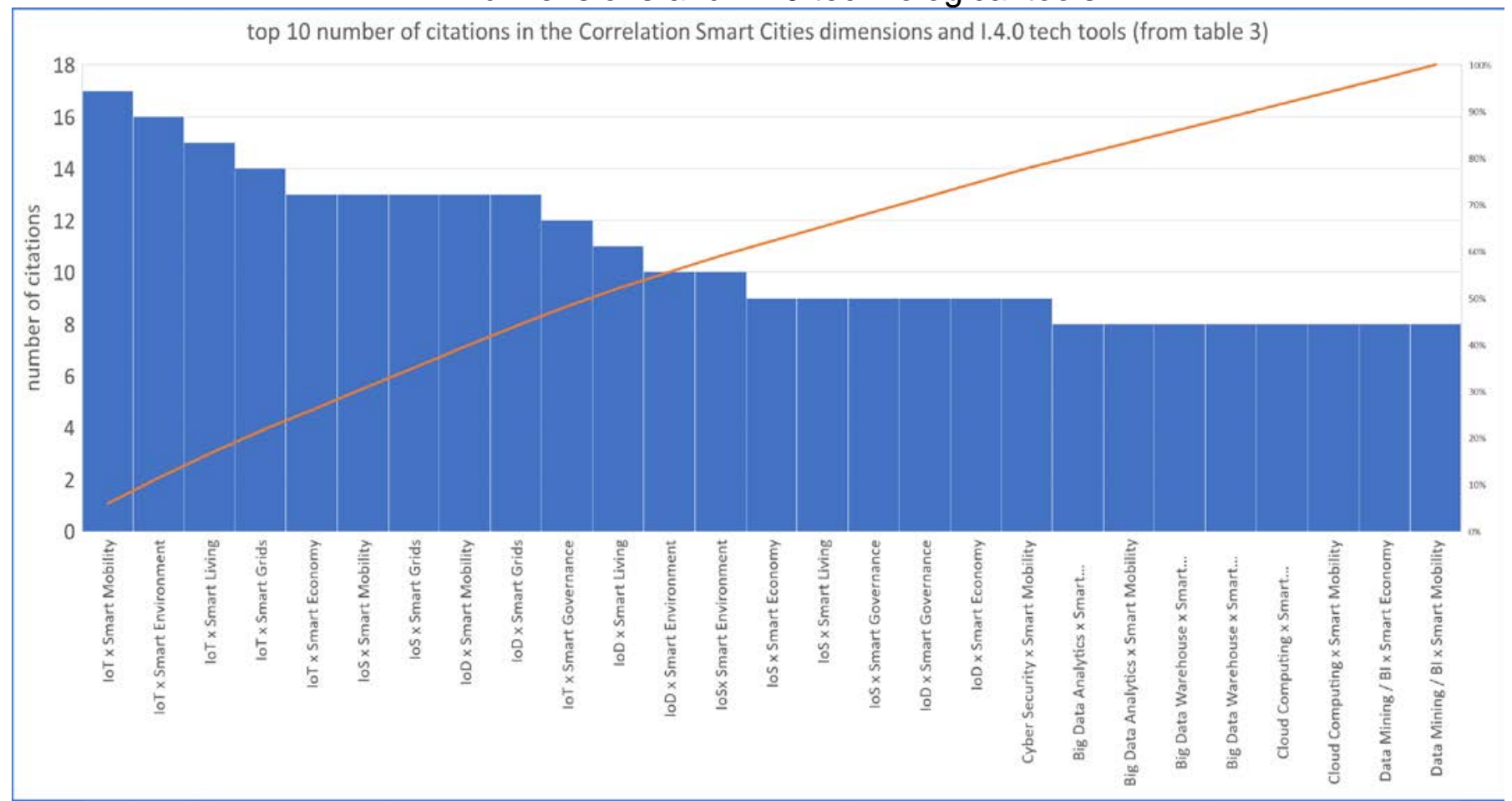

Source: Storolli, Makiya and Cesar, 2017

\section{CONCLUSION AND LIMITATION}

The bibliographical study was limited to articles related to the subject "Industry 4.0", "Smart City", scientific articles (congresses and magazines), technical (nonscientific) magazines, Government publications and consulting available. Due to the contemporaneity of the themes, there is an opportunity for further detailed studies due to the fast-technological tools development.

This scenario stimulates the exploration of the Smart Cities as living laboratory with increasingly decentralized operations, due to the connectivity 
INDEPENDENT JOURNAL OF MANAGEMENT \& PRODUCTION (IJM\&P)

http://www.ijmp.jor.br

v. 10, n. 3, May - June 2019

ISSN: 2236-269X

DOI: 10.14807/ijmp.v10i3.792

provided by technology, with changes in real time, more autonomously, in the same way as in the industry 4.0 approach.

With the need for a careful and systematic analysis in the adoption of systems and processes for the new configurations of cities, many people can be affected directly or indirectly by this living laboratory.

The disruptive models of managing services for smart citizens are embedded in an innovation ecosystem that increasingly demands ICT management, in which the knowledge-based and connected society prevails in information as a groundwork.

Therefore, Big Data Warehouse \& Analytics, Cloud Computing, Cyber Security, Data Mining, BI and Smart Devices become the basis of all technological change allowing and stimulating other tools such as IOT, IOD, IOS in achieving full growth and development in a scalable way.

When scalability is achieved, these technological solutions become more affordable, more cost-effective, more democratically available, which amplifies and increases the capillarity of Triple Helix (companies, government agencies and researchers) actions to improve quality of life of people in general.

Following the original purpose of this article, it was identified the relations of the Smart Cities development dimensions with Industry 4.0 technological tools and their relevance as main booster for a "new" Smart Cities concept, and it was released a management guidance proposal for a strategic development orientation of a "new Smart City", taking priority with IoT, IoD and IoS.

\section{REFERENCES}

AHMED, E.; YAQOOB, I.; GANI, A.; IMRAN, M.; GUIZANI, M. (2016) Internet-ofThings-Based Smart Environments: State of the Art, Taxonomy, and Open Research Challenges. Enabling Wireless Communications and Networking Technologies for the Internet of Things, IEEE Wireless Communications, October, p. 10-16.

AHVENNIEMI, H.; HUOVILA, A.; PINTO-SEPPÄ, I.; AIRAKSINEN, M. (2017) What are the differences between sustainable and smart cities? Cities, v. 60, n. A, p. 234245.

ANDERL, R. (2014) Industrie 4.0 - Advanced Engineering of Smart Products and Smart Production. ResearchGate, Conference Paper from October 2014. (Online) Available from: https://www.researchgate.net/publication/270390939 (Accessed: August $24^{\text {th }} 2016$ ) 
INDEPENDENT JOURNAL OF MANAGEMENT \& PRODUCTION (IJM\&P)

http://www.ijmp.jor.br

v. 10, n. 3, May - June 2019

ISSN: 2236-269X

DOI: 10.14807/ijmp.v10i3.792

ANTHOPOULOS, L. (2016) Smart utopia VS smart reality - 10 smart city cases. Cities, v. 63, p. 128-148.

BADII, C.; BELLINI, P.; CENNI, D.; DIFINO, A.; NESI, P.; PAOLUCCI, M. (2017) Analysis and assessment of a knowledge based smart city architecture providing service APIs. Future Generation Computer Systems, v. 75, n. 14-29.

BAKICI, T.; ALMIRALL, E.; WAREHAM, J. (2013) A smart city initiative: The case of Barcelona. Journal of the Knowledge Economy, v. 4, n. 2, p. 135-148.

BANKS, J. (1998) Handbook of simulation: principles, methodology, advances, applications, and practice.

BASIRI, M.; AZIM, A. Z.; FARROKHI, M. (2017) Smart City Solution for Sustainable Urban Development. European Journal of Sustainable Development, v. 6, n. 1, p. 71-84.

BROOKS, R. A. (1991) Artificial Intelligence - Intelligence without representation.

CARAGLIU, A.; DEL BO, C.; NIJKAMP, P. (2009; 2011) Smart cities in Europe. 3rd Central European Conference in Regional Science - CERS, p. 45-59.

CEBREIROS, J.; GULÍN, M. P. (2014) Guia Smart Cities - Cidades com futuro, Agenda Digital local, Spain, 1ª Edição, p. 1-150.

COCCHIA, A. (2014) Smart and Digital City: A systematic literature review. In DAMERI, R. P.; ROSENTHAL-SABROUX, C. (Eds.), Smart City, p. 13-43.

EUROPEAN SMART CITIES 4.0 (2015) Available from: http://www.smartcities.eu/?cid=2\&ver=4 (Accessed: November $5^{\text {th }}$ '2017)

FLEICH, E. (2010) What is the Internet of Things? Auto-ID Labs.

GAUR, A.; SCOTNEY, B.; PARR, G.; MCCLEAN, S. (2015) Smart City Architecture and its Applications based on IoT. Procedia Computer Science, v. 52, p. 10891094.

GERLITZ, L. (2015) Design for product and service innovation in Industry 4.0 and emerging smart society. Journal of Security and Sustainability, v. 5, n. 2, p. 181198.

GLOBAL CITIES INSTITUTE-GCI, (2015) Cities and sustainable infrastructure. GCIF Policy snapshot $n^{\circ} 3$, Cities and Sustainable Infrastructure Series, n. 1, p. 1-40.

GRAHAM, S.; MARVIN, S. (2001) Splintering urbanism: Networked infrastructures, technological Mobilities and the urban conditions. Routledge, $1^{\text {st }}$ edition, p. 1-481.

HERMANN, M.; TOBIAS, P.; BORIS, O. (2015) Design Principles for Industrie 4.0 Scenarios: A Literature Review.

HOPEN, J. (2015) 7 características importantes para diferenciar BI, Data Mining e Big Data. Available from: https://aquare.la/7-caracteristicas-importantes-paradiferenciar-bi-data-mining-e-big-data/ (Accessed: September 19'th '2017)

KAGERMANN, H.; WAHLSTER, W.; HELBIG, J. (2013) Recommendations for implementing the strategic initiative INDUSTRIE 4.0. acatech-National Academy of Science and Engineering, p. 1-82. 
INDEPENDENT JOURNAL OF MANAGEMENT \& PRODUCTION (IJM\&P)

http://www.ijmp.jor.br

v. 10, n. 3, May - June 2019

ISSN: 2236-269X

DOI: 10.14807/ijmp.v10i3.792

KARAKOSE, M.; YETIS, H. (2017) A Cyberphysical System Based Mass-

Customization Approach with Integration of Industry 4.0 and Smart City. Hindawi

Wireless Communications and Mobile Computing, v. 2017, n. 9, p. 1-10.

KOURTIT, K.; NIJKAMP, P. (2012) Smart cities in the innovation age. IGI Global, $1^{\text {st }}$ edition: $1-660$

KOURTIT, K.; NIJKAMP, P.; STEENBRUGGEN, J. (2017) The significance of digital data systems for smart city policy. Socio-Economic Planning Sciences, v. 58, p. 13-21.

KUMMITHA, R. K. R.; CRUTZEN, N. (2017) How do we understand smart cities? Cities, v. 67, p. 43-52.

KYLILI, A.; FOKAIDES, P. A. (2015) European smart cities: The role of zero energy buildings.

LJAZ, S.; SHAH, M. A.; KHAN, A.; AHMED, M. (2016) Smart Cities: A Survey on Security Concerns. IJACSA-International Journal of Advanced Computer Science and Applications, v. 7, n. 2, p. 1-14.

LOM, M.; PRIBYL, O.; SVITEK, M. (2016) Industry 4.0 as a Part of Smart Cities. Smart Cities Symposium Prague, p. 1-6.

MACHADO, R. T. M. (2000) Rastreabilidade, Tecnologia da Informação e coordenação de sistemas Agroindustriais.

MAGLIO, P. P.; LIM, C. H. (2016) Innovation and Big Data in Smart Service Systems.

MAIER, S. (2016) Smart energy systems for smart city districts: case study Reininghaus District. Maier Energy, Sustainability and Society, p. 6-23.

MARTIN, M. (2015) Building the impact economy: Our future, yea or nay. Springer International Publishing Switzerland, p. 1-209.

MARTÍNEZ-BARBERÁ, H.; HERRERO-PÉREZ, D. (2009) Autonomous navigation of an automated guided vehicle in industrial environments.

MASEK, P.; MASEK, J.; FRANTIK, P.; FUJDIAK, R.; OMETOV, A.; HOSEK, J.; REEV, S.; MLYNEK, P.; MISUREC, J. (2016) A Harmonized Perspective on

Transportation Management in Smart Cities: The Novel loT-Driven Environment for Road Traffic Modeling.

MELL, P.; GRANCE T. (2011) The NIST definition of cloud computing.

METZNER, V.; SILVA, R.; CUGNASCA, C. (2014) Modelo de rastreabilidade de medicamentos utilizando identificação por radiofrequência, redes de sensores sem fio e o conceito de Internet das Coisas, ANPET.

MILOSLAVSKAYA, N.; TOLSTOY, A. (2016) Big Data, Fast Data and Data Lake Concepts.

MINOLI, D.; SOHRABY, K.; OCCHIOGROSSO, B. (2017) IOT Considerations, Requirements, and Architectures for Smart Buildings-Energy Optimization and NextGeneration Building Management Systems. IEEE Internet Of Things Journal, v. 4, n. 1, p. 269-283.

PARASURAMAN, R.; SHERIDAN, T. B.; WICKENS C. D. (2000) A Model for Types and Levels of Human Interaction with Automation. 
INDEPENDENT JOURNAL OF MANAGEMENT \& PRODUCTION (IJM\&P)

http://www.ijmp.jor.br

v. 10, n. 3, May - June 2019

ISSN: 2236-269X

DOI: 10.14807/ijmp.v10i3.792

PRYTULA, L. (2011) Sensor inteligente.

ROBLEK, V.; MEŠKO, M.; KRAPEŽ, A. (2016) A Complex View of Industry 4.0.

SAGE Open, April-June, p. 1-11.

RUSSOM, P. (2011) Big Data Analytics.

SAMPRI, A.; MAVRAGANI, A.; TSAGARAKIS, K. P. (2016) Evaluating Google Trends as a Tool for Integrating the 'Smart Health' Concept in the Smart Cities' Governance in USA.

SANDERSON, D. (2010) Programming Google App Engine: Build and Run Scalable Web Apps on Google's Infrastructure, O'Reilly Media.

SCHUMACHER, A.; EROLB, S.; SIHNA, W. (2016) A maturity model for assessing Industry 4.0 readiness and maturity of manufacturing enterprises.

SCHWAB, K. (2016) A Quarta Revolução Industrial - World Economic Forum. SOLMS, R. V.; NIEKERK, J. V. (2013) From information security to cyber security.

TALARI, S.; SHAFIE-KHAH, M.; SIANO, P.; LOIA, V.; TOMMASETTI, A.; CATALÃO, J. P. S. (2017) A Review of Smart Cities Based on the Internet of Things Concept. Energies, v. 10, n. 421, p. 1-23.

THOMPSON, C. W. (2005) Smart devices and soft controllers, IEEE Internet Computing.

VERMESAN, O.; FRIESS, P. (2014) Internet of Things-From Research and Innovation to Market Deployment. River Publishers Denmark: p. 1-374, accessed in October 26 ${ }^{\text {th }} 2017$ on <https://books.google.com.br/books?hl=pt-

$B R \& \mid r=\& i d=r H Y G Z 0 w x L P 0 C \& o i=f n d \& p g=P R 1 \& d q=i n d u s t r y+4.0+a n d+s m a r t+c i t y \& o t s$ $=N Y C r q V s B D I \& s i g=1 c s Z 4 f A g R \times G r m H x V r f L H R i v c Q Z A \# v=o n e p a g e \& q \& f=f a l s e>$

ZHOU, K.; ZHOU, T. L. L. (2015) Industry 4.0: Towards Future Industrial Opportunities and Challenges. 12th International Conference on Fuzzy Systems and Knowledge Discovery (FSKD), p. 2147-2152. 